\title{
A New Hilbert-type Integral Inequality with the Homogeneous Kernel of Real Degree Form and the Integral in Whole Plane
}

\author{
Xie Zitian ${ }^{1}$ K. Raja Rama Gandhi ${ }^{2}$ Zheng Zeng $^{3}$ \\ ${ }^{1}$ Department of Mathematics, Zhaoqing University, Zhaoqing, Guangdong, China 526061 \\ ${ }^{2}$ Resource person in Oxford University, India and BITS-Vizag, INDIA. \\ ${ }^{3}$ Department of Mathematics, Shaoguan University, Shaoguan, Guangdong, China512005
}

Keywords: Hilbert-type integral inequality, weight function, $\mathrm{H}$ older's inequality, function.

Abstract: In this paper,we build a new Hilbert's inequality with the homogeneo is kernel real order and the integral in whole plane. The equivalent inequality is considered. best con ant factor is calculated using $\Psi$ f unction.

\section{INTRODUCTION}

If $f(x), g(x) \geq 0$, such that $0<\int_{0}^{\infty} f^{2}(x) \mathrm{d} x<\infty \quad 0<\int_{0}^{\infty} g^{2}(x)$

$$
\int_{0}^{\infty} \int_{0}^{\infty} \frac{f(x) g(y)}{x+y} \mathrm{~d} x \mathrm{~d} y<\pi\left(\int_{0}^{\infty} f^{2}(x) \mathrm{d} x\right)^{1 / 2}\left(\int_{0}^{\infty} g^{q}(x) \mathrm{d} x\right)^{1 / 2}
$$

where the constant factor $\pi$ is the best possible. Ineque ty (1.1) is vell-known as Hilbert's integral inequality, which has been extended by Hardy-Riesz as If $p>1,1 / p+1 / q=1 f(x), g(x) \geq 0$; such that $0<\int_{0} \quad x<\infty$, and $0<\int_{0}^{\infty} g^{q}(x) \mathrm{d} x<\infty$; then we have the following Hardy-Hilbert's inte rarm lity:

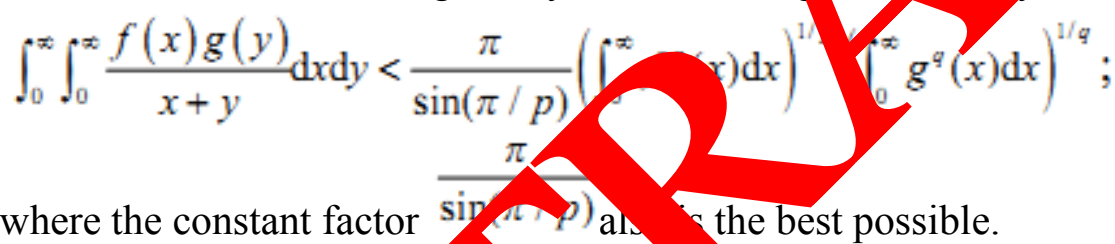

Hilbert's inequality attrac on attentio recent years.Actually, inequalities (1.1)and(1.2)

have many generaliza ns à. variations. (1.1) has been strengthened by Yang and others (including double s/10s inequali $43,4,6-21]$.

In 2008, Zitian $X$ e and heng Zen, gave a new Hilbert-type Inequality [4] as follows

If $a>0, b>0, c>p-1,1 /,+1 / q=1 f(x), g(x) \geq 0$; such that

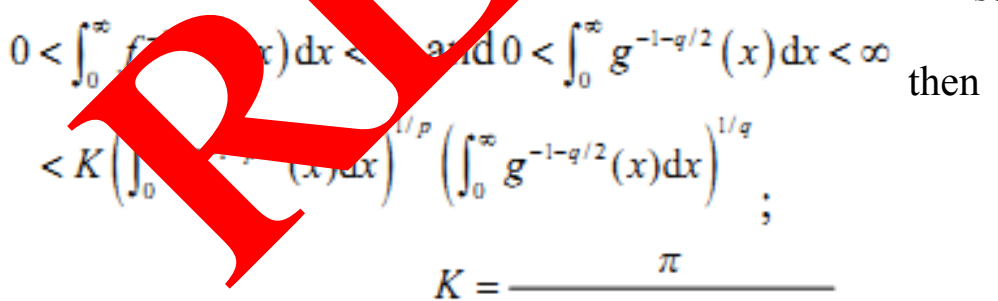

where the constant factor $K=\overline{(a+b)(a+c)(c+b)}$ is the best possible.

In 2010,Jianhua Xhong and Bicheng Yang gave a new Hilbert-type Inequality [5] as follows :

Assume that

$\lambda, p>0(p \neq 1), r>1,1 / p+1 / q=1,1 / r+1 / s=1, \phi(x)=x^{p\left(1-\frac{\lambda}{r}\right)-1}, \varphi(x)=x^{q\left(1-\frac{\lambda}{s}\right)-1}, x \in(0, \infty)$,
$K=\Gamma(\beta+1) \sum_{k=0}^{\infty}(-1)^{k}\left(\begin{array}{l}\alpha-\lambda \\ k\end{array}\right)\left[\frac{1}{(k+\lambda / r)^{\beta+1}}+\frac{1}{(k+\lambda / s)^{\beta+1}}\right]$, and $f, g \geq 0$, 
$0<\|f\|_{p, \phi}:=\left\{\int_{0}^{\infty} x^{p(1-\lambda / r)-1} f^{p}(x) \mathrm{d} x\right\}^{1 / p}<\infty, 0<\|g\|_{q, \varphi}<\infty$ then

(1) for $p>1$ we have the following equivalent inequalities:

$\int_{0}^{\infty} \int_{0}^{\infty} \frac{|\ln (x / y)|^{\beta} f(x) g(y)}{|x-y|(\max \{|x|,|y|\})^{\alpha}} \mathrm{d} x \mathrm{~d} y<K\|f\|_{p, \phi}\|g\|_{q, \varphi}$

(2)For $0<\mathrm{p}<1$ the reverse of (1.5) with the best constant factor $\mathrm{K}$.

$\int_{0}^{\infty} \int_{0}^{\infty} \frac{|\ln (x / y)|^{\beta} f(x) g(y)}{|x-y|(\max \{|x|,|y|\})^{\alpha}} \mathrm{d} x \mathrm{~d} y>K\|f\|_{p, \phi}\|g\|_{q, \varphi}$

The main purpose of this paper is to build a new Hilbert-type inequality with the neous kernel of real order and the integral in whole plane, by estimating the weight $f$ inction us $\Psi$ function. The equivalent inequality is considered

We knew that (in this paper, $\gamma$ is the Euler's constant.) $\psi(z)=\frac{\Gamma^{\prime}(z)}{\Gamma(z)}=-\gamma+\sum_{n=0}^{\infty}\left(\frac{1}{n+1}-\frac{1}{n+z}\right), \psi(1)=-\gamma, \psi\left(\frac{1}{2}\right)=-\gamma-2 \ln 2$.

Recent XIE Zitin and ZHOU Qinghua prove that the expression $c$ the $\Psi-\mathrm{h}$ ion admits a finite expression in elementary function for rational number $z$, and pro e

$\psi\left(\frac{a}{b}\right)=\Gamma^{\prime}\left(\frac{a}{b}\right) / \Gamma\left(\frac{a}{b}\right)=-\ln b-\gamma-\ln 2-\frac{\pi}{2} \cot \frac{a \pi}{b}+\sum_{k=1}^{b-1} \cos \frac{2 k \sigma}{b} \sin \frac{k \pi}{b}$

and have

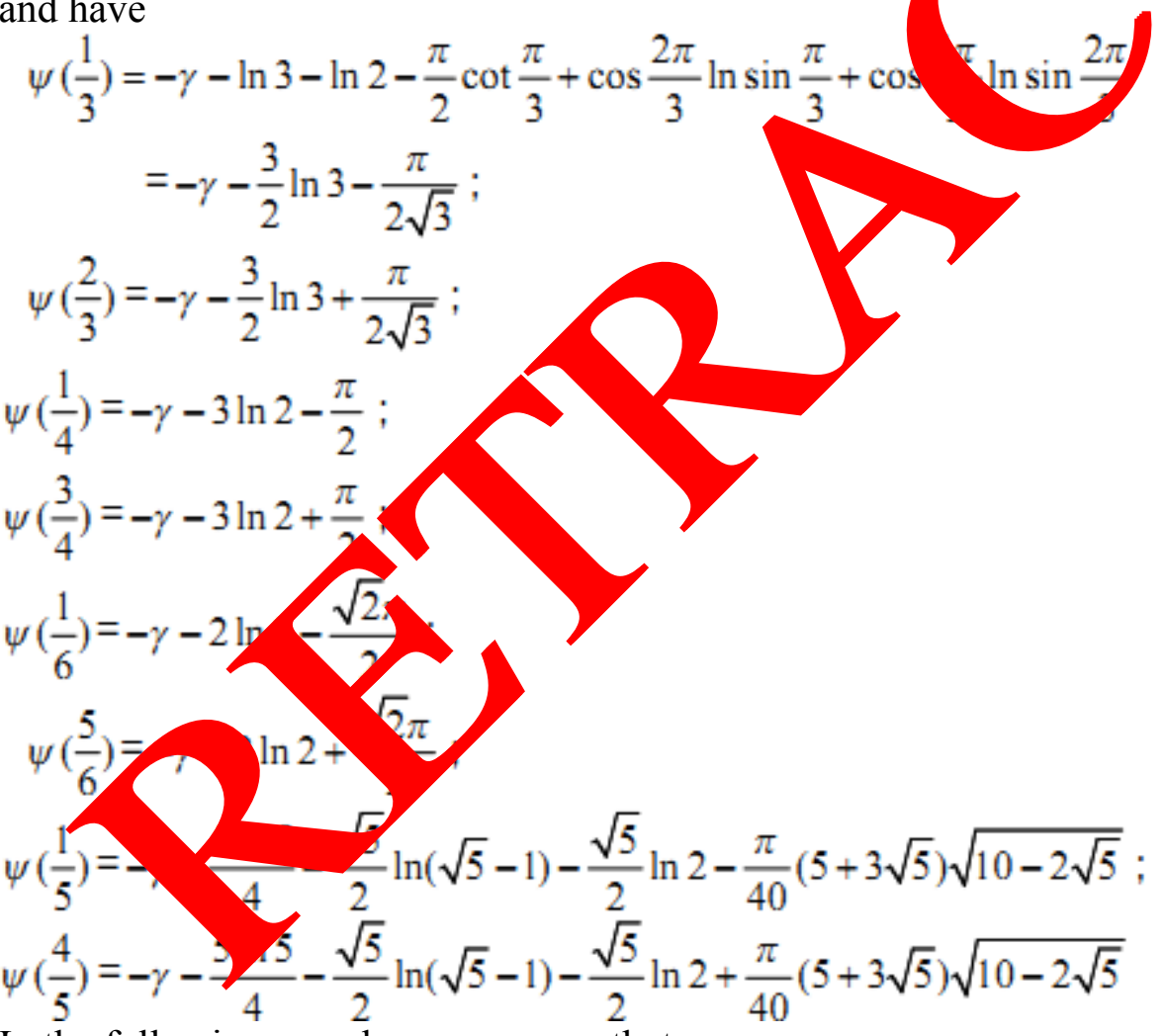

In the following, we always suppose that:

$1 / p+1 / q=1, p>1, \min \{a \lambda+b \mu, a \mu+b \lambda\}>-1, a \mu+b \lambda \neq 0, a \lambda+b \mu \neq 0, \mu>0, \lambda>0$.

$a+b=1$.

\section{SOME LEMMAS}

We start by introducing some Lemmas.

Lemma 2.1 If $s>0, r \neq 0, r>-s$, then 
1) $\int_{0}^{1} x^{r-1} \ln \left(1-x^{s}\right) d x=-\frac{1}{r}\left[\gamma+\psi\left(\frac{r+s}{s}\right)\right]$

2) $\int_{0}^{1} x^{r-1} \ln \left(1+x^{s}\right) d x=\frac{1}{r} \ln 2-\frac{1}{2 r}\left[\psi\left(\frac{r+2 s}{2 s}\right)-\psi\left(\frac{r+s}{2 s}\right)\right]$

Proof. we obtain,'

1) $-\int_{0}^{1} x^{r-1} \ln \left(1-x^{s}\right) d x=\int_{0}^{1} x^{r-1} \sum_{l=1}^{\infty} \frac{x^{l s}}{l} d x$

$=\sum_{l=1}^{\infty} \int_{0}^{l} \frac{x^{l s+r-1}}{l} d x=\sum_{l=1}^{\infty} \frac{1}{l(r+l s)}$

$=\frac{1}{r} \sum_{l=1}^{\infty}\left(\frac{1}{l}-\frac{1}{l+r / s}\right)$

$=\frac{1}{r}\left[\gamma+\psi\left(\frac{r+s}{s}\right)\right]$

2) $\int_{0}^{1} x^{r-1} \ln \left(1+x^{s}\right) d x=\int_{0}^{1} x^{r-1} \sum_{l=1}^{\infty}(-1)^{l-1} \frac{x^{l s}}{l} d x$

$=\sum_{l=1}^{\infty} \int_{0}^{1}(-1)^{l-1} \frac{x^{l s+r-1}}{l} d x=\sum_{l=1}^{\infty} \frac{(-1)^{l-1}}{l(r+l s)}$

$=\lim _{N \rightarrow \infty}\left\{\frac{1}{r} \sum_{l=1}^{2 N+1}(-1)^{l-1} \frac{1}{l}-\frac{1}{2 r}\left[\sum_{n=0}^{N}\left(\frac{1}{n+1}-\frac{1}{n+\frac{r+2 s}{2}}\right)-\sum_{n=0}^{N}\left(\frac{1}{+1}-\frac{1}{n+\frac{l}{2 s}}\right)\right]\right\}$

$=\frac{1}{r} \ln 2-\frac{1}{2 r}\left[\psi\left(\frac{r+2 s}{2 s}\right)-\psi\left(\frac{r+s}{2 s}\right)\right]$

The lemma is proved.

In particular, if $r>-1, r \neq 0$, then

$$
\begin{aligned}
& \int_{0}^{1} u^{r-1} \ln \frac{u^{2}+u+1}{u^{2}-u+1} d u \\
& =\int_{0}^{1} u^{r-1} \ln \left(1-u^{3}\right) d y \int_{0}^{-1} \ln \left(1-\quad d u-\left[\int_{0}^{1} u^{r-1} \ln \left(1+u^{3}\right) d u-\int_{0}^{1} u^{r-1} \ln (1+u) d u\right]\right. \\
& =\frac{1}{r}\left[\psi(r+1)-\psi+3-\frac{1}{2 y} \psi\left(\frac{r+6}{6}\right)-\psi\left(\frac{r+3}{6}\right)-\psi\left(\frac{r+2}{2}\right)+\psi\left(\frac{r+1}{2}\right)\right] \\
& \left(y \operatorname{lng} \varphi(x+1)=\psi(x)+\frac{1}{x}\right) \\
& \left.=\frac{1}{r}[\psi(r)-\psi / 3)\right]+\frac{1}{2 r}\left[\psi\left(\frac{r}{6}\right)-\psi\left(\frac{r+3}{6}\right)-\psi\left(\frac{r}{2}\right)+\psi\left(\frac{r+1}{2}\right)\right] \\
& =\frac{1}{r}\left[\psi(r)-\psi\left(\frac{r}{3}\right)\right]+\frac{1}{2 r}\left[2 \psi\left(\frac{r}{6}\right)-\psi\left(\frac{r}{6}\right)-\psi\left(\frac{r+3}{6}\right)-2 \psi\left(\frac{r}{2}\right)+\psi\left(\frac{r}{2}\right)+\psi\left(\frac{r+1}{2}\right)\right] \\
& \text { (using } \psi\left(x+\frac{1}{2}\right)+\psi(x)=2 \psi(2 x)-\psi\left(\frac{r+1}{2}\right) \\
& =\frac{2}{r}\left[\psi(r)-\psi\left(\frac{r}{3}\right)\right]+\frac{1}{r}\left[\left(\frac{r}{6}\right)-\psi\left(\frac{r}{2}\right)\right]
\end{aligned}
$$


Lemma 2.2 Define the weight functions as follow:

$$
\begin{aligned}
& w(x):=\int_{-\infty}^{\infty} \frac{|x|^{a(\mu-\lambda)}}{|y|^{1-b(\mu-\lambda)}} \frac{(\min \{|x|,|y|\})^{\lambda}}{(\max \{|x|,|y|\})^{\mu}\left|\ln \frac{x^{2}+x y+y^{2}}{x^{2}+y^{2}}\right| d y,} \\
& \tilde{w}(y):=\int_{-\infty}^{\infty} \frac{|y|^{b(\mu-\lambda)}}{|x|^{1-a(\mu-\lambda)}} \frac{(\min \{|x|,|y|\})^{\lambda}}{(\max \{|x|,|y|\})^{\mu}\left|\ln \frac{x^{2}+x y+y^{2}}{x^{2}+y^{2}}\right| d x,}
\end{aligned}
$$

Then

$$
\begin{aligned}
& w(x)=\tilde{w}(y) \\
& =\frac{2}{a \lambda+b \mu}\left[\psi(a \lambda+b \mu)-\psi\left(\frac{a \lambda+b \mu}{3}\right)\right]+\frac{1}{a \lambda+b \mu}\left[\psi\left(\frac{a \lambda+b \mu}{6}\right)-\psi\left(\frac{a \lambda+b \mu}{2}\right)\right] \\
& +\frac{2}{a \mu+b \lambda}\left[\psi(a \mu+b \lambda)-\psi\left(\frac{a \mu+b \lambda}{3}\right)\right]+\frac{1}{a \lambda+b \mu}\left[\psi\left(\frac{a \mu+b \lambda}{6}\right)-\psi\left(\frac{a \mu+b \lambda}{2}\right)\right] \\
& :=k
\end{aligned}
$$

Proof We only prove that $w(x)=k$ for $x \in(-\infty, 0)$

Using lemma 2.1, setting $\mathrm{y}=\mathrm{ux}$, and $\mathrm{y}=-\mathrm{ux}$

$$
\begin{aligned}
w(x) & :=\int_{-\infty}^{0} \frac{(-x)^{a(\mu-\lambda)}}{(-y)^{1-b(\mu-\lambda)}} \frac{(\min \{(-x),(-y)\})^{\lambda}}{(\max \{(-x),(-y)\})^{\mu}} \ln \frac{x^{2}+x y+y^{2}}{x^{2}+y^{2}} d y \\
& \quad+\int_{0}^{\infty} \frac{(-x)^{a(\mu-\lambda)}}{y^{1-b(\mu-\lambda)}} \frac{(\min \{(-x), y\})^{\lambda}}{(\max \{(-x), y\})^{\mu}} \ln \frac{x^{2}}{x^{2}+y^{2}} \frac{y^{2}}{1+y^{2}} d y \\
& :=w_{1}+w_{2}
\end{aligned}
$$

Then

$$
\begin{aligned}
& w_{1}=\int_{0}^{1} u^{-1+a \lambda+b \mu} \ln \frac{u^{2}+u+1}{u^{2}+1} d u+\int_{1}^{\infty} u^{-1-a \lambda-b \mu} \ln \frac{u}{u}+u^{2}-a y \\
& =\int_{0}^{1} u^{-1+a \lambda+b \mu} \ln \frac{u^{2}+u+1}{u^{2}+1} d u+\int_{0}^{1}-1+a \mu-b \frac{u^{2}+u}{u^{2}} d u \\
& w_{2}=\int_{0}^{1} u^{-1+a \lambda+b \mu} \ln \frac{u^{2}+1}{u^{2}-u} \quad u+\int_{1}^{\infty} u^{-} \quad \ln \frac{u^{2}+1}{u^{2}-u+1} d u \\
& =\int_{0}^{1} u^{-1+a \lambda+b \mu} \ln \frac{u^{2}+1}{-u+1} d u+\int_{0}-1+a \mu+b \lambda \ln \frac{u^{2}+1}{u^{2}-u+1} d u \\
& \text { And } \quad w_{1}+\int_{0}^{1} u \int_{b \mu} \ln \frac{y}{u^{2}-u+1} d u+\int_{0}^{1} u^{-1+a \mu+b \lambda} \ln \frac{u^{2}+u+1}{u^{2}-u+1} d u \\
& \left.=\frac{2}{a \lambda+b}-\psi\left(\frac{a \lambda+b \mu}{3}\right)\right]+\frac{1}{a \lambda+b \mu}\left[\psi\left(\frac{a \lambda+b \mu}{6}\right)-\psi\left(\frac{a \lambda+b \mu}{2}\right)\right] \\
& +\frac{2}{a \mu+b \lambda}\left[y(a \mu+b \lambda)-\psi\left(\frac{a \mu+b \lambda}{3}\right)\right]+\frac{1}{a \lambda+b \mu}\left[\psi\left(\frac{a \mu+b \lambda}{6}\right)-\psi\left(\frac{a \mu+b \lambda}{2}\right)\right] \text {. } \\
& =k
\end{aligned}
$$

Similarly, setting $x=y / u$, and $x=-y / u$

$$
\begin{aligned}
\tilde{w}(y)=\int_{-\infty}^{0} & \frac{(-y)^{b(\mu-\lambda)}}{(-x)^{1-a(\mu-\lambda)}} \frac{(\min \{(-x),(-y)\})^{\lambda}}{(\max \{(-x),(-y)\})^{\mu}} \ln \frac{x^{2}+x y+y^{2}}{x^{2}+y^{2}} d x \\
& +\int_{0}^{\infty} \frac{y^{b(\mu-\lambda)}}{(-x)^{1-\alpha(\mu-\lambda)}} \frac{(\min \{(-x), y\})^{\lambda}}{(\max \{(-x), y\})^{\mu}} \ln \frac{x^{2}+y^{2}}{x^{2}+x y+y^{2}} d x
\end{aligned}
$$




$$
\begin{aligned}
= & \int_{-\infty}^{0} \frac{(-y)^{b(\mu-\lambda)}}{(-y / u)^{1-a(\mu-\lambda)}} \frac{(\min \{(-y / u),(-y)\})^{\lambda}}{(\max \{(-y / u),(-y)\})^{\mu}} \ln \frac{(y / u)^{2}+(y / u) y+y^{2}}{(y / u)^{2}+y^{2}} d(y / u) \\
& +\int_{0}^{\infty} \frac{y^{b(\mu-\lambda)}}{(y / u)^{1-a(\mu-\lambda)}} \frac{(\min \{(y / u), y\})^{\lambda}}{(\max \{(y / u), y\})^{\mu}} \ln \frac{(-y / u)^{2}+y^{2}}{(-y / u)^{2}+(-y / u) y+y^{2}} d(-y / u) \\
= & \int_{0}^{\infty} u^{-1+b(\mu-\lambda)} \frac{(\min \{1, u\})^{\lambda}}{\left(\max \{1, u)^{\mu}\right.} \ln \frac{u^{2}+u+1}{u^{2}+1} d u+\int_{0}^{\infty} u^{-1+b(\mu-\lambda)} \frac{(\min \{1, u\})^{\lambda}}{\left(\max \{1, u)^{\mu}\right.} \ln \frac{u^{2}+1}{u^{2}-u+1} d u \\
= & w_{1}+w_{2}=k
\end{aligned}
$$

and the lemma is proved.

Lemma 2.3 For $\varepsilon>0$; and $\min \{a \mu+b \lambda-2 \varepsilon / q, a \lambda+b \mu-2 \varepsilon / q\}>-1$, define bo functions, $\tilde{g}$ as follow:

$$
\begin{gathered}
\tilde{f}(x)=\left\{\begin{array}{llc}
x^{a(\mu-\lambda)-1-2 \varepsilon / p}, & \text { if } & x \in(1, \infty), \\
0, & \text { if } & x \in[-1,1], \\
(-x)^{a(\mu-\lambda)-1-2 \varepsilon / p}, & \text { if } & x \in(-\infty,-1),
\end{array}\right. \\
\tilde{g}(x)=\left\{\begin{array}{llc}
x^{b(\mu-\lambda)-1-2 \varepsilon / q}, & \text { if } & x \in(1, \infty), \\
0, & \text { if } & x \in[-1,1], \\
(-x)^{b(\mu-\lambda)-1-2 \varepsilon / q}, & \text { if } & x \in(-\infty,-1),
\end{array}\right.
\end{gathered}
$$

Then

$$
\begin{aligned}
& I(\varepsilon):=\left.\varepsilon\left\{\int_{-\infty}^{\infty}|x|^{p[1-a(\mu-\hat{\lambda})]-1} \widetilde{f}^{p}(x) d x\right\}^{1 / p}\left\{\int_{-\infty}^{\infty}|x|^{q[1-b(\mu-\hat{\lambda})]}-\alpha\right) d x\right|^{1 / \rho}=1 ; \\
& \tilde{I}(\varepsilon):=\varepsilon \int_{-\infty}^{\infty} \int_{-\infty}^{\infty} \tilde{f}(x) \tilde{g}(y) \frac{(\min \{|x|,|y|\})^{\lambda}}{\left(\max \{|x|,|,| l \mid)^{\mu}\right.}\left|\ln \frac{\lambda}{-x+y^{2}}\right|^{-x} d x d y \rightarrow k\left(\varepsilon \rightarrow 0^{+}\right)
\end{aligned}
$$

Proof Easily

$$
I(\varepsilon)=\varepsilon\left\{2 \int_{1}^{\infty} x^{-1} x^{-2 \varepsilon} d x\right\}
$$

Let $\mathrm{y}-\mathrm{Y}$, using $\tilde{f}(-x)=(x)-x)=\vec{g}(x)$ and

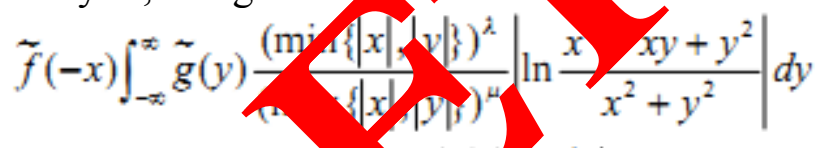

$$
=f(Y) \frac{\{|x|,|Y|\})^{2}}{(\operatorname{man}|x|,|Y|\})^{\mu}}\left|\ln \frac{x^{2}+x Y+Y^{2}}{x^{2}+Y^{2}}\right| d Y
$$

we have that $f(x) \int_{-\infty} g(y) \frac{(\min \{|x|,|y|\})^{\lambda}}{(\max \{|x|,|y|\})^{\mu}}\left|\ln \frac{x^{2}+x y+y^{2}}{x^{2}+y^{2}}\right| d y \quad$ is an even function on $\mathrm{x}$, , then

$$
\begin{aligned}
& \tilde{I}(\varepsilon):=2 \varepsilon \int_{-\infty}^{\infty} \tilde{f}(x)\left(\int_{-\infty}^{\infty} \tilde{g}(y) \frac{(\min \{|x|,|y|\})^{\lambda}}{(\max \{|x|,|y|\})^{\mu}}\left|\ln \frac{x^{2}+x y+y^{2}}{x^{2}+y^{2}}\right| d y\right) d x \\
& =2 \varepsilon\left[\int_{1}^{\infty} x^{a(\mu-\lambda)-1-2 \varepsilon / p}\left(\int_{-\infty}^{-1}(-y)^{b(\mu-\lambda)-1-2 \varepsilon / q} \frac{(\min \{|x|,|y|\})^{\lambda}}{(\max \{|x|,|y|\})^{\mu}}\left|\ln \frac{x^{2}+x y+y^{2}}{x^{2}+y^{2}}\right| d y\right) d x\right. \\
& \left.+\int_{1}^{\infty} x^{a(\mu-\hat{\lambda})-1-2 \varepsilon / p}\left(\int_{1}^{\infty} y^{b(\mu-\hat{\lambda})-1-2 \varepsilon / q} \frac{(\min \{|x|,|y|\})^{\lambda}}{(\max \{|x|,|y|\})^{\mu}}\left|\ln \frac{x^{2}+x y+y^{2}}{x^{2}+y^{2}}\right| d y\right) d x\right] \\
& :=I_{1}+I_{2}
\end{aligned}
$$


Setting $y=t x$ then

$$
\begin{aligned}
& I_{1}=2 \varepsilon \int_{1}^{\infty} x^{a(\mu-\lambda)-1-2 \varepsilon / p}\left(\int_{1}^{\infty} y^{b(\mu-\lambda)-1-2 \varepsilon / q} \frac{(\min \{x, y\})^{\lambda}}{(\max \{x, y\})^{\mu}} \ln \frac{x^{2}+y^{2}}{x^{2}-x y+y^{2}} d y\right) d x \\
& =2 \varepsilon \int_{1}^{\infty} x^{-1-2 \varepsilon}\left(\int_{1 / x}^{\infty} t^{b(\mu-\lambda)-1-2 \varepsilon / q} \frac{(\min \{1, t\})^{\lambda}}{(\max \{1, t\})^{\mu}} \ln \frac{t^{2}+1^{2}}{t^{2}-t+1^{2}} d t\right) d x \\
& =2 \varepsilon \int_{1}^{\infty} x^{-1-2 \varepsilon}\left(\int_{1}^{\infty} t^{b(\mu-\hat{\lambda})-1-2 \varepsilon / q} \frac{(\min \{1, t\})^{\lambda}}{(\max \{1, t\})^{\mu}} \ln \frac{t^{2}+1^{2}}{t^{2}-t+1^{2}} d t\right) d x \\
& +2 \varepsilon \int_{1}^{\infty} x^{-1-2 \varepsilon}\left(\int_{1 / x}^{1} t^{b(\mu-\hat{\lambda})-1-2 \varepsilon / q} \frac{(\min \{1, t\})^{\lambda}}{(\max \{1, t\})^{\mu}} \ln \frac{t^{2}+1^{2}}{t^{2}-t+1^{2}} d t\right) d x \\
& =\int_{1}^{\infty} t^{b(\mu-\hat{\lambda})-1-2 \varepsilon / q} \frac{(\min \{1, t\})^{\lambda}}{(\max \{1, t\})^{\mu}} \ln \frac{t^{2}+1^{2}}{t^{2}-t+1^{2}} d t \\
& +2 \varepsilon \int_{0}^{1} t^{b(\mu-\lambda)-1-2 \varepsilon / q} \frac{(\min \{1, t\})^{\lambda}}{(\max \{1, t\})^{\mu}} \ln \frac{t^{2}+1^{2}}{t^{2}-t+1^{2}}\left(\int_{1 / t}^{\infty} x^{-1-2 \varepsilon} d x\right) d t \\
& =\int_{1}^{\infty} t^{b(\mu-\lambda)-1-2 \varepsilon / q} \frac{(\min \{1, t\})^{\lambda}}{(\max \{1, t\})^{\mu}} \ln \frac{t^{2}+1^{2}}{t^{2}-t+1^{2}} d t \\
& +\int_{0}^{1} t^{b(\mu-\lambda)-1-2 \varepsilon / p} \frac{(\min \{1, t\})^{\lambda}}{(\max \{1, t\})^{\mu}} \ln \frac{t^{2}+1^{2}}{t^{2}-t+1^{2}} d t \\
& =\int_{0}^{\infty} t^{b(\mu-\hat{\lambda})-1-2 \varepsilon / q} \frac{(\min \{1, t\})^{\lambda}}{(\max \{1, t\})^{\mu}} \ln \frac{t^{2}+1^{2}}{t^{2}-t+1^{2}} d t \\
& +\int_{0}^{1}\left(t^{2 \varepsilon / p}-t^{-2 \varepsilon / q}\right) t^{b(\mu-\lambda)-1} \frac{(\min \{1, t\})^{\lambda}}{(\max \{1, t\})^{\mu}} \ln \frac{t^{2}+1}{t^{2}-t+1}
\end{aligned}
$$

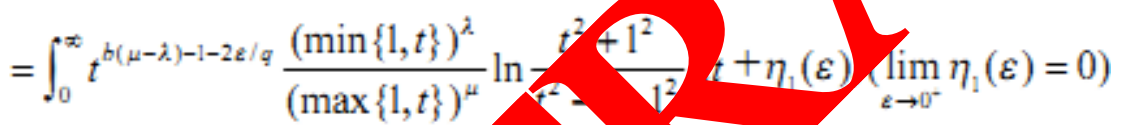

Similarly

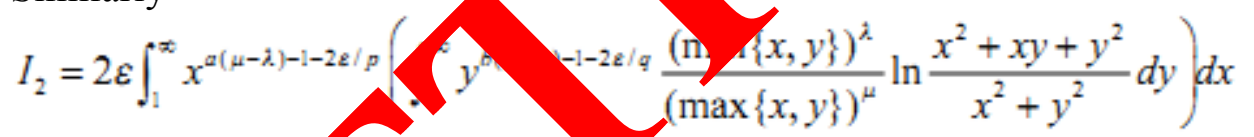

$$
=\int_{0}^{\infty} t^{b(\mu-}-\frac{\left.\operatorname{in}\{1, t\})^{\lambda}\right)}{(\max \{t\})^{\mu}} \ln \frac{t^{2}+t+1^{2}}{t^{2}+1^{2}} d t+\eta_{2}(\varepsilon),\left(\lim _{\varepsilon \rightarrow 0^{-}} \eta_{2}(\varepsilon)=0\right)
$$

by lemma are ha

$$
\begin{aligned}
& \tilde{I}(\varepsilon) \\
& +\frac{1}{a \lambda+b \mu+b \mu-2 \varepsilon / q}\left[\psi\left(\frac{a \lambda+b \mu-2 \varepsilon / q}{6}\right)-\psi\left(\frac{a \lambda+b \mu-2 \varepsilon / q}{2}\right)\right] \\
& +\frac{2}{a \mu+b \lambda-2 \varepsilon / q}\left[\psi(a \mu+b \lambda-2 \varepsilon / q)-\psi\left(\frac{a \mu+b \lambda-2 \varepsilon / q}{3}\right)\right] \\
& +\frac{1}{a \mu+b \lambda-2 \varepsilon / q}\left[\psi\left(\frac{a \mu+b \lambda-2 \varepsilon / q}{6}\right)-\psi\left(\frac{a \mu+b \lambda-2 \varepsilon / q}{2}\right)\right]+\eta_{1}(\varepsilon)+\eta_{2}(\varepsilon)
\end{aligned}
$$

we know that $\psi(x)$ is a continuous function, then $\lim _{\varepsilon \rightarrow 0^{+}} \tilde{I}(\varepsilon)=k$

The lemma is proved. 
Lemma 2.4 If $\mathrm{f}(\mathrm{x})$ is a nonnegative measurable function, and 0 $<\int_{-\infty}^{\infty}|x|^{p[1-a(\mu-\lambda)]-1} f^{p}(x) d x<\infty$

Then

$$
\begin{aligned}
J & :=\int_{-\infty}^{\infty}|y|^{p b(\mu-\lambda)-1}\left(\int_{-\infty}^{\infty} f(x) \frac{(\min \{|x|,|y|\})^{\lambda}}{(\max \{|x|,|y|\})^{\mu}}\left|\ln \frac{x^{2}+x y+y^{2}}{x^{2}+y^{2}}\right| d x\right)^{p} d y \\
& \leq k^{p} \int_{-\infty}^{\infty}|x|^{p[1-\alpha(\mu-\lambda)]-1} f^{p}(x) d x
\end{aligned}
$$

Proof By lemma 2.2,we find

$$
\begin{aligned}
& \left(\int_{-\infty}^{\infty} f(x) \frac{(\min \{|x|,|y|\})^{i}}{(\max \{|x|,|y|\})^{\mu}}\left|\ln \frac{x^{2}+x y+y^{2}}{x^{2}+y^{2}}\right| d x\right)^{p} \\
& =\left(\int_{-\infty}^{\infty} \frac{(\min \{|x|,|y|\})^{\lambda}}{(\max \{|x|,|y|\})^{\mu}}\left|\ln \frac{x^{2}+x y+y^{2}}{x^{2}+y^{2}}\right|\left(\frac{|x|^{[1-a(\mu-\lambda)]^{\prime} q}}{|y|^{[1-b(\mu-\lambda)]^{\prime / p}}} f(x)\right)\left(\frac{|y|^{[1-b(\mu-\lambda)]^{\prime / p}}}{|x|^{[1-a(\mu-\lambda)]^{\prime / q}}}\right)\right. \\
& \leq \int_{-\infty}^{\infty} \frac{(\min \{|x|,|y|\})^{\lambda}}{(\max \{|x|,|y|\})^{\mu}}\left|\ln \frac{x^{2}+x y+y^{2}}{x^{2}+y^{2}}\right| \frac{|x|^{[1-a(\mu-\lambda)](p-1)}}{|y|^{1-b(\mu-\lambda)}} f^{p}(x) d x \\
& \times\left(\int_{-\infty}^{\infty} \frac{(\min \{|x|,|y|\})^{\lambda}}{(\max \{|x|,|y|\})^{\mu}}\left|\ln \frac{x^{2}+x y+y^{2}}{x^{2}+y^{2}}\right| \frac{|y|^{[1-b(\mu-\lambda)](q-1)}}{|x|^{1-a(\mu-\lambda)}}\right. \\
& =k^{p-1}|y|^{1-p b(\mu-\lambda)} \int_{-\infty}^{\infty} \frac{(\min \{|x|,|y|\})^{\lambda}}{\left(\max \{|x|,|y|)^{\mu}\right.}\left|\ln \frac{x^{2}+x y+y^{2}}{x^{2}+y^{2}}\right| \frac{|x|^{[1-\alpha(\mu-\lambda)}}{|-y|^{1-b(\mu-\lambda)}}-g(x) d x \\
& \left.J \leq k^{p-1} \int_{-\infty}^{\infty}\left[\int_{-\infty}^{\infty} \frac{(\min \{|x|,|y|\})^{\lambda}}{(\max \{|x|,|y|\})^{\mu}}\left|\ln \frac{x^{2}+x y+y^{2}}{x^{2}+y^{2}}\right| \frac{|x|^{[1-x \mid}}{|y|^{1-b}}-f^{2}\right) d x\right] d y
\end{aligned}
$$

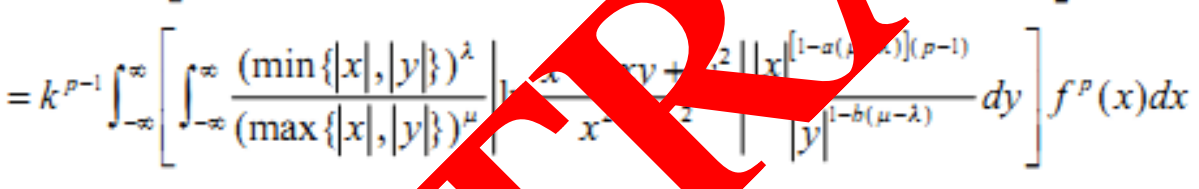

$$
\begin{aligned}
& =k^{p} \int_{-\infty}^{\infty}|x|^{p[1-a(\mu-\lambda)]-1} f^{p} d d x \\
& 3 \text { MAIN RESV TS }
\end{aligned}
$$

Theorem 3.1 If $\mathrm{p}$; both unctions, $\mathrm{f}(\mathrm{x})$ and $\mathrm{g}(\mathrm{x})$, are nonnegative measurable functions, and

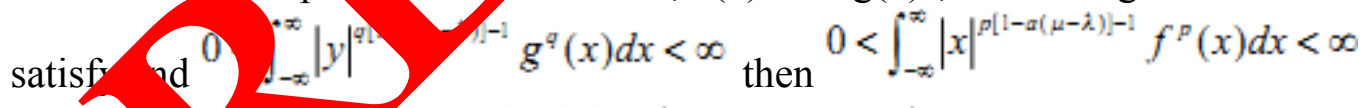

$$
\begin{aligned}
I^{*}:= & \left.\int_{-\infty}\right) \\
& <k\left(\int_{-\infty}^{\infty}|x|^{p[1-a(\mu-\lambda)]-1} f^{p}(x) d x\right)^{1 / p}\left(\int_{-\infty}^{\infty}|x|^{q[1-b(\mu-\lambda)]-1} g^{q}(x) d x\right)^{1 / q}
\end{aligned}
$$

And

$$
\begin{aligned}
& J=\int_{-\infty}^{\infty}|y|^{p b(\mu-\lambda)-1}\left(\int_{-\infty}^{\infty} f(x) \frac{(\min \{|x|,|y|\})^{\lambda}}{\left(\max \{|x|,|y| \xi)^{\mu}\right.}\left|\ln \frac{x^{2}+x y+y^{2}}{x^{2}+y^{2}}\right| d x\right)^{p} d y \\
& <k^{p} \int_{-\infty}^{\infty}|x|^{p[1-a(\mu-\lambda)]-1} f^{p}(x) d x
\end{aligned}
$$

Inequalities (3.1)and (3.2) are equivalent,and where the constant factors $\mathrm{k}$ and $\mathrm{kp}$ arethe best possible. 
Proof If there exist a $y \in(-\infty, 0) \cup(0, \infty)$,such that (2.7) takes the form of equality, then there exists constants $\mathrm{M}$ and $\mathrm{N}$, such that they are not all zero, and

$M \frac{|x|^{[1-a(\mu-\lambda)](p-1)}}{|y|^{1-b(\mu-\lambda)}} f^{p}(x)=N \frac{|y|^{[1-b(\mu-\lambda)](q-1)}}{|x|^{1-a(\mu-\lambda)}}$ a.e. In $(-\infty, \infty)$

Hence, there exists a constant $C$, such that

$M|x|^{p[1-a(\mu-\lambda)]} f^{p}(x)=N|y|^{q[1-b(\mu-\lambda)]}=C$ a.e. $\operatorname{In}(-\infty, \infty)$

It means that $\mathrm{M}=0$. In fact, if $M \neq 0$, then

$|x|^{[[1-\alpha(\mu-\hat{\lambda})]-1} f^{p}(x)=\frac{C}{M|x|}$ a.e. $\operatorname{In}(-\infty, \infty)$

which contradicts the fact that $0<\int_{-\infty}^{\infty}|x|^{p[1-a(\mu-\lambda)]-1} f^{p}(x) d x<\infty$

0 : This is too a contradiction and hence by (2.7), we have (3.2). By Holder's ineauality th veight and (3.2), we have,

$I^{*}:=\int_{-\infty}^{\infty}\left[|y|^{b(\mu-\lambda)-\frac{1}{p}} \int_{-\infty}^{\infty} f(x) \frac{(\min \{|x|,|y|\})^{\lambda}}{(\max \{|x|,|y|\})^{\mu}}\left|\ln \frac{x^{2}+x y+y^{2}}{x^{2}+y^{2}}\right| d x\right][\mid$

$\leq J^{1 / p}\left(\int_{-\infty}^{\infty}|y|^{q[1-b(\mu-\hat{k})]-1} g^{q}(y) d y\right)^{1 / q}$

Using (3.2), we have (3.1).

Setting

$g(y)=|y|^{p b(\mu-\lambda)-1}\left(\int_{-\infty}^{\infty} f(x) \frac{(\min \{|x|,|y|\})^{\lambda}}{(\max \{|x|,|y|\})^{\mu}} \mid \ln \right.$

Then

$J=\int_{-\infty}^{\infty}|y|^{q[1-b(\mu-\lambda)]^{-1}} g^{q}(y) d y$ by (2 we h $\mathrm{e}^{J<\infty}$ if $\mathrm{J}=0$ then (3.2) is proved;

If $0<J<\infty$ we obtain

$0<\int_{-\infty}^{\infty}|y|^{q[1-b(\mu-\lambda)]^{-1}} g^{q}(y)=J=I^{*}$

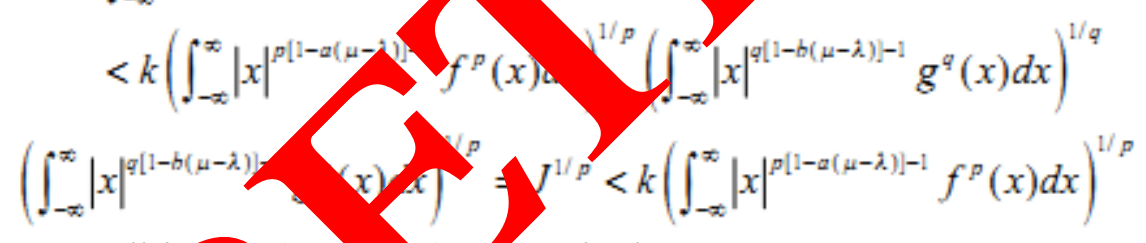

Inequaliti and a e equivalent.

If the onstai factor $\mathrm{k} A(3.1)$ is not the best possible, then there exists a positive $\mathrm{h}$ (with $\mathrm{h}<$ $\mathrm{k}), \mathrm{such}$

$$
\begin{aligned}
& \int_{-\infty}^{\infty} \int(x) g(y) \frac{(\min \{|x|,|y|\})^{\lambda}}{(\max \{|x|,|y|\})^{\mu} \mid}\left|\ln \frac{x^{2}+x y+y^{2}}{x^{2}+y^{2}}\right| d x d y \\
& <h\left(\int_{-\infty}^{\infty}|x|^{p[1-\alpha(\mu-\lambda)]-1} f^{p}(x) d x\right)^{1 / p}\left(\int_{-\infty}^{\infty}|x|^{q[1-b(\mu-\lambda)]-1} g^{q}(x) d x\right)^{1 / q}
\end{aligned}
$$

For $\varepsilon>0$ by (2.5), using lemma 2.3 ,we have

$$
k+o(1)<\varepsilon h\left(\int_{-\infty}^{\infty}|x|^{p[1-a(\mu-\lambda)]-1} \tilde{f}^{p}(x) d x\right)^{1 / p}\left(\int_{-\infty}^{\infty}|x|^{q[1-b(\mu-\lambda)]-1} \tilde{g}^{q}(x) d x\right)^{1 / q}=h
$$

Hence we find, $\mathrm{k}+\mathrm{o}(1)<\mathrm{h}$ : For ${ }^{\varepsilon \rightarrow 0^{-}}$it follows that $k \leq h$ which contradicts the fact that $\mathrm{h}<\mathrm{k}$. Hence the constant $\mathrm{k}$ in (3.1) is the best possible.

Thus we complete the prove of the theorem. 
Theorem 3.2 If $1>p>0$; both functions, $\mathrm{f}(\mathrm{x})$ and $\mathrm{g}(\mathrm{x})$, are nonnegative measurable functions, and satisfy

$$
\begin{gathered}
0<\int_{-\infty}^{\infty}|x|^{p[1-a(\mu-\lambda)]-1} f^{p}(x) d x<\infty \text { and } 0<\int_{-\infty}^{\infty}|x|^{q[1-b(\mu-\lambda)]-1} g^{q}(x) d x<\infty \\
\int_{-\infty}^{\infty} \int_{-\infty}^{\infty} f(x) g(y) \frac{(\min \{|x|,|y|\})^{\lambda}}{(\max \{|x|,|y|\})^{\mu}}\left|\ln \frac{x^{2}+x y+y^{2}}{x^{2}+y^{2}}\right| d x d y \\
>k\left(\int_{-\infty}^{\infty}|x|^{p[1-a(\mu-\lambda)]-1} f^{p}(x) d x\right)^{1 / p}\left(\int_{-\infty}^{\infty}|x|^{q[1-b(\mu-\lambda)]-1} g^{q}(x) d x\right)^{1 / q} \\
J>k^{p} \int_{-\infty}^{\infty}|x|^{p[1-a(\mu-\lambda)]-1} f^{p}(x) d x
\end{gathered}
$$

And

$$
\begin{aligned}
L & :=\int_{-\infty}^{\infty}|x|^{q \alpha(\mu-\lambda)-1}\left(\int_{-\infty}^{\infty} \frac{(\min \{|x|,|y|\})^{\lambda}}{(\max \{|x|,|y|\})^{\mu}}\left|\ln \frac{x^{2}+x y+y^{2}}{x^{2}+y^{2}} g(y)\right| d y\right)^{q} d x \\
& <k^{q} \int_{-\infty}^{\infty}|y|^{q[1-b(\mu-\lambda)]-1} g^{q}(y) d y
\end{aligned}
$$

Inequalities (3.5),(3.6)and (3.7) are equivalent, and where the ons factors $\mathrm{k} \mathrm{K}^{\mathrm{p}}$ and $\mathrm{k}^{\mathrm{q}}$ are the best possible

Proof By the reverse Holder's inequality and the same way, we can an the reverseforms of (2.7)and (3.3). And then we deduce the (3.5), by the som way,we obtain (3.6).

Setting $\mathrm{g}(\mathrm{y})$ as the theorem 1 , we obtain $\mathrm{J}>0$, if $J=\infty$, by (3.5)

$$
\begin{aligned}
& \quad \int_{-\infty}^{\infty}|y|^{q[1-b(\mu-\lambda)]-1} g^{q}(y) d y=J=I^{*} \\
& >k\left(\int_{-\infty}^{\infty}|x|^{p[1-a(\mu-\lambda))-1} f^{p}(x) d x\right)^{1 / p}\left(\int_{-\infty}^{\infty} \mid x x^{g[1-b(\mu-\lambda)]-1} \&(x) d x\right)^{\prime} \\
& \text { and we have (3.6), and inequalitie 5)ar (3.6) ar equivalent. }
\end{aligned}
$$

Setting

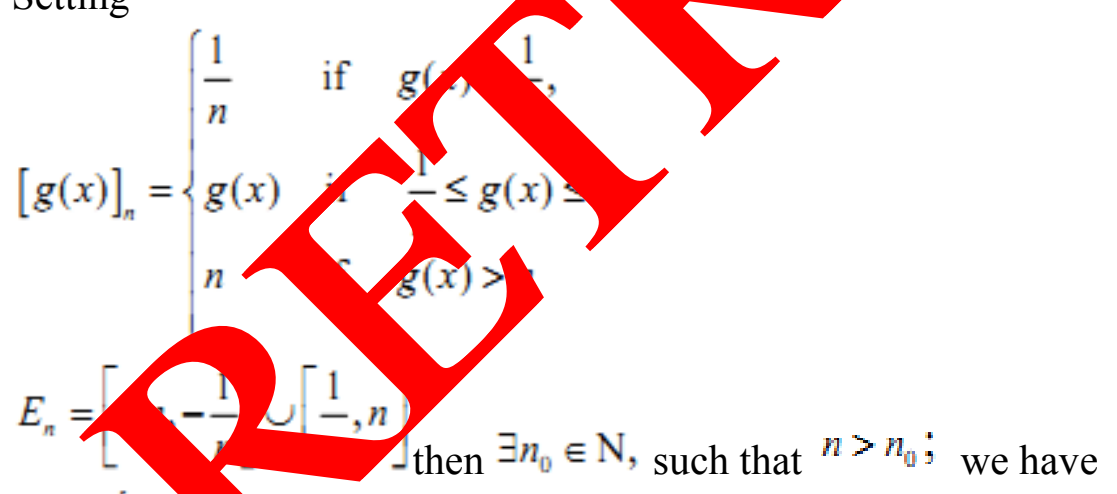

$\int_{E_{n}}|y|^{q[1-b(\mu)}$
And

$$
\begin{gathered}
{[f(x)]_{n}=|x|^{g(\mu-\lambda)-1}\left(\int_{E_{n}}[g(x)]_{n} \frac{\left(\min \{|x|,|y|)^{\lambda}\right.}{\left(\max \{|x|,|y| \xi)^{\mu}\right.}\left|\ln \frac{x^{2}+x y+y^{2}}{x^{2}+y^{2}}\right| d y\right)^{q-1}} \\
{[L(x)]_{n}=\int_{E_{n}}|x|^{q a(\mu-\lambda)-1}\left(\int_{E_{n}}[g(x)]_{n} \frac{(\min \{|x|,|y|\})^{\lambda}}{(\max \{|x|,|y|\})^{\mu}}\left|\ln \frac{x^{2}+x y+y^{2}}{x^{2}+y^{2}}\right| d y\right)^{q} d x}
\end{gathered}
$$

Then $\exists n_{0} \in \mathrm{N}$, such that $n>n_{0} ;$ we have

$\int_{E_{n}}|y|^{q[1-b(\mu-\lambda)]-1} g^{q}(y) d y<\infty$, 
In particular, from (4.1) we get the following particular cases:

1)If $\lambda+\mu=4$; then $k=2\left[\psi(2)-\psi\left(\frac{2}{3}\right)\right]+\frac{2}{3}\left[\psi\left(\frac{1}{3}\right)-\psi(1)\right]=2+\frac{3 \ln 3}{2}-\frac{\sqrt{3} \pi}{2}$, we have

$$
\int_{-\infty}^{\infty} \int_{-\infty}^{\infty} f(x) g(y) \frac{(\min \{|x|,|y|\})^{\lambda}}{(\max \{|x|,|y|\})^{4-\lambda}}\left|\ln \frac{x^{2}+x y+y^{2}}{x^{2}+y^{2}}\right| d x d y
$$

$<\left(2+\frac{3 \ln 3}{2}-\frac{\sqrt{3} \pi}{2}\right)\left(\int_{-\infty}^{\infty}|x|^{p(\lambda-1)-1} f^{p}(x) d x\right)^{1 / p}\left(\int_{-\infty}^{\infty}|x|^{q(\lambda-1)-1} g^{q}(x) d x\right)^{1 / q}$

2) If $\lambda+\mu=3$; then $k=\frac{8}{3}\left[\psi\left(\frac{3}{2}\right)-\psi\left(\frac{1}{2}\right)\right]+\frac{4}{3}\left[\psi\left(\frac{1}{4}\right)-\psi\left(\frac{3}{4}\right)\right]=\frac{4}{3}(4-\pi)$

$\int_{-\infty}^{\infty} \int_{-\infty}^{\infty} f(x) g(y) \frac{(\min \{|x|,|y|\})^{\lambda}}{(\max \{|x|,|y|\})^{4-\lambda}}\left|\ln \frac{x^{2}+x y+y^{2}}{x^{2}+y^{2}}\right| d x d y$

$<\left(2+\frac{3 \ln 3}{2}-\frac{\sqrt{3} \pi}{2}\right)\left(\int_{-\infty}^{\infty}|x|^{p(\lambda-1)-1} f^{p}(x) d x\right)^{1 / p}\left(\int_{-\infty}^{\infty}|x|^{q(\lambda-1)-1} g^{q}(x) d x\right)$

3) If $\lambda+\mu=2$; then $k=4\left[\psi(1)-\psi\left(\frac{1}{3}\right)\right]+2\left[\psi\left(\frac{1}{6}\right)-\psi\left(\frac{1}{2}\right)\right]$

$$
\int_{-\infty}^{\infty} \int_{-\infty}^{\infty} f(x) g(y) \frac{(\min \{|x|,|y|\})^{\lambda}}{(\max \{|x|,|y|\})^{2-\lambda}}\left|\ln \frac{x^{2}+x y+y^{2}}{x^{2}+y^{2}}\right| d y
$$

$<\left(6 \ln 3+\frac{2 \pi}{\sqrt{3}}-\sqrt{2} \pi\right)\left(\int_{-\infty}^{\infty}|x|^{p \lambda-1} f^{p}(x) d x\right)^{1 / p}$

B) Let $\lambda=\mu$ in (3.1), then we have a 1ntegral 1n aality with the homogeneous kernel of 0 degree form as follows:

$$
\begin{aligned}
& \int_{-\infty}^{\infty} \int_{-\infty}^{\infty} f(x) g(y)\left(\frac{\min \{|x|,|y|\}}{\max \{|x|} \frac{x^{2}-x y+y^{2} \mid}{-+y} \mid y d y\right. \\
& <k(\lambda)\left(\int_{-\infty}^{\infty}|x|^{p-1} \rho(\lambda)\right)^{1 / p}\left(\int_{-\infty}^{\infty} \mid \sum g^{q}(x) d x\right)^{1 / q} \\
& \left.k(\lambda)=\frac{4}{2}\left[(\lambda)-\left(\frac{\lambda}{3}\right)\right]+\frac{\lambda}{\lambda} \psi\left(\frac{\lambda}{6}\right)-\psi\left(\frac{\lambda}{2}\right)\right]
\end{aligned}
$$

There

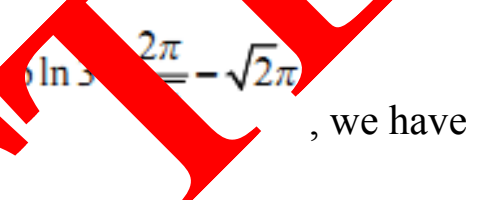

\section{Refere ces}

[1] Haro Cambridge

[2] Hardy G.) A., Note on a theorem of Hilbert concerning series of positive terms, Proceedings London Math. Soc., 1925,23(2):45-46.

[3] Zitian Xie and Zheng Zeng, A Hilbert-type integral inequality whose kernel is a homogeneous form of degree-331

[4] Zitian Xie, Zheng Zeng, A Hilbert-type integral inequality with a non- homogeneous form and a best constant factor, Advances and Applications in Mathematical Sciens 2010,3(1),61-71

[5] Jianhua Xhong and Bicheng Yang, On a relation to four basic Hilbert-type integral inequalities, Applied Mathematical Sciences,Vol. 4, 2010,no,19,923-930. 
[6] XIE Zitian and ZHOU Qinghua,A note on a Property of psi -function, Wuhan University Journal of Natural Sciences, Wuhan University Journal of Natural Science, 2011,16(2),139-142.

[7] Bicheng Yang, On a Hilber-type integral inequality with a combination kerne and applications, Kyungpook Mathematical Journal, 2010(50),281-288

[8] Zitian Xie , Zheng Zeng, The Hilbert-type integral inequality with the system Kernel of $-\lambda$ degree homogeneous form, Kyungpook Mathematical Journal, 2010(50),297-306

[9] Xie Zitian, A New Hilbert-type integral inequality with the homogeneous kernel of real numberdegre, Journal of Jishou University (Natural Science Edition), 2011,32(4)26-30

[10] Zitian Xie and Bicheng Yang,A new Hilbert-type integral inequality with some narameters and its reverse,Kyungpook Mathematical Journal,2008,(48):93-100

[11] Zitian Xie,A new Hilbert-type inequality with the kernel of -30 -homogenes Iournal or ilin University (Science edition),2007,45(3):369-373.

[12] Zitian Xie and Juming Murong,A rewerse Hilbert-type inequ ity parameters, Journal of Jilin University (Science edition), 2008,46 (4):665-

[13] Zitian Xie,A new reverse Hilbert-type inequality with a b constan cter, J.Math.Appl, 2008,(343):1154-1160.

[14] Zheng Zeng and Zi-tian Xie, A Hilbert's inequality a bes. nstant factor, Journal of Inequalities and Applications, 2009, Article ID 820176, pages doi:10.1,$/ 2009 / 820176$

[15] Xie Zitian ,Zeng Zheng,On generality of Hilbert's equality wi h best constant factor, Natural Science Journal of Xiangtan University, 2010, 32(3) : 1

[16] Zheng Zeng and Zitian Xie, On a new "1hert-type gral inequality with the integral in whole plane, Journal of Inequalities and Applic tion 2010, Article ID 256796, 8 pages, 2010. doi:10.1155/2010/256796

[17] Zitian Xie,Bicheng Yang, Z eng eng, A New Hilbert-type integral inequality with the homogeneous kernel of real nber legree, ournal of Jilin University(Science Edition), 2010,48(6) : 941-945.

[18] Zitian Xie and Fano Zhou, A the best constant factor, Journal f Sic o Norma University (Natural Science) 2009, 32(5): 626-629

[19] Xie Zitian,Zerg Zneng, A H K-type integral inequality with the integral in whole plane and the kernel of -4 gree b mogeneoas form, i-manager's Journal on Mathematics, Vol. 1 No. 3, Julyseptember 2012,0

[20] Ziti ie,Zhe Ze,g,Qinghua Zhou, A new Hilbert-type integral inequality with the homo neous kernel of real number-degree and its equivalent inequality forms,Journal of Jilin Univers s ition), 2012,50(4), 693-697.

[21] Xie $\lambda$.Zeng Zheng, A new half-discrete Hilbert-type inequality with the homogeneous kernel of degr $-4 \mu$, Journal of Jishou University(Natural Science Edition), 2012,33 (2), 15-19 